\title{
MOVES RELATING C-COMPLEXES: A CORRECTION TO CIMASONI'S "A GEOMETRIC CONSTRUCTION OF THE CONWAY POTENTIAL FUNCTION"
}

\author{
CHRISTOPHER W. DAVIS, TAYLOR MARTIN, AND CAROLYN OTTO
}

\begin{abstract}
In groundbreaking work from 2004, Cimasoni gave a geometric computation of the multivariable Conway potential function in terms of a generalization of a Seifert surface for a link called a C-complex 2. Lemma 3 of that paper provides a family of moves which relates any two C-complexes for a fixed link. This allows for an approach to studying links from the point of view of C-complexes and in following papers it has been used to derive invariants. This lemma is false. We present counterexamples, a correction with detailed proof, and an analysis of the consequences of this error on subsequent works that rely on this lemma.
\end{abstract}

\section{Introduction AND STATEMENT OF RESUlts}

A C-complex is a generalization of a Seifert surface to the setting of links, or more broadly, colored links. Specifically, a C-complex is a union of compact, oriented, embedded surfaces in $S^{3}$ which are allowed to intersect in clasps with no triple points. These C-complexes first appeared in work of Cooper [9, 10] where they were used to compute the Alexander module, signature, and nullity of a 2-component link. In 2004, Cimasoni extended Cooper's work to compute the multivariable Conway potential function for colored links of arbitrarily many components [2]. In subsequent works including [4, [5], 6], 7], and [8, this is extended to other signatures, Alexander modules, Casson-Gordon invariants, Blanchfield forms, splitting numbers, links in quasi-cylinders, and other topics.

A colored link is an oriented link $L$ in $S^{3}$ such that each component $K$ of $L$ is assigned a color $\sigma(K) \in\{1, \ldots, n\}$. We will express an $n$-colored link as $(L, \sigma)$ or $L=L_{1} \cup \cdots \cup L_{n}$ where $L_{i}=\sigma^{-1}(\{i\})$ is the $i$-colored sublink. Thus, a 1-colored link is just an oriented link, and an $m$-colored, $m$-component link is an ordered, oriented link. Two $n$-colored links $(L, \sigma)$ and $\left(L^{\prime}, \sigma^{\prime}\right)$ are isotopic if there exists a color and orientation preserving ambient isotopy from $L$ to $L^{\prime}$. A C-complex $F=F_{1} \cup \cdots \cup F_{n}$ is said to be bounded by the colored link $L$ if $\partial F_{i}=L_{i}$ for $i=1, \ldots, n$. In [2] Cimasoni provides a set of geometric moves on C-complexes which relate any pair of C-complexes for isotopic links.

Lemma 1.1 (Lemmas 2 and 3 in [2]. See also Lemma 2.2 in [5]). Let $F$ and $F^{\prime}$ be C-complexes bounded by isotopic colored links. Then, $F$ and $F^{\prime}$ can be transformed into each other by a finite number of the following operations and their inverses:

(T0) Ambient isotopy,

(T1) Handle attachment on one surface,

(T2) Addition of a ribbon intersection followed by a "push along an arc" through this intersection, as in Figure 1 a,

Date: May 24, 2021.

2020 Mathematics Subject Classification. 57K10. 


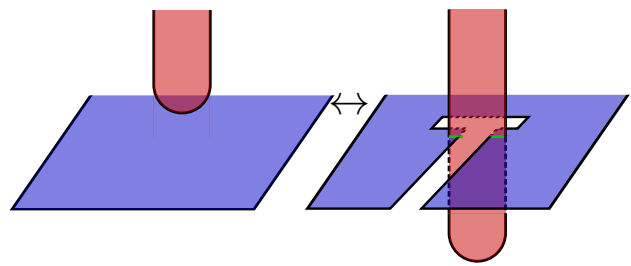

(a) The (T2) move

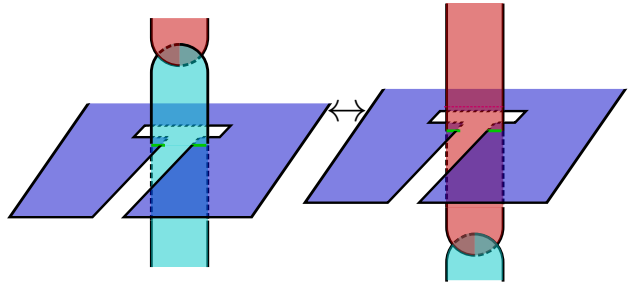

(b) The (T3) move

FiguRe 1

(T3) The pass through a clasp move, as in Figure 1 .

Some noteworthy applications of this lemma are in [2], [5], and [6] where these C-complex moves are used to verify that certain properties of a C-complex are invariants of the underlying link. The first goal of this document is to demonstrate that the set of moves in Lemma 1.1 is incomplete.

Theorem 1.2. The C-complexes $F$ and $F^{\prime}$ appearing in Figures $2 b$ and $2 c$ cannot be related by any sequence of the moves (T0), (T1), (T2), and (T3). Therefore, Lemma 1.1 is false.

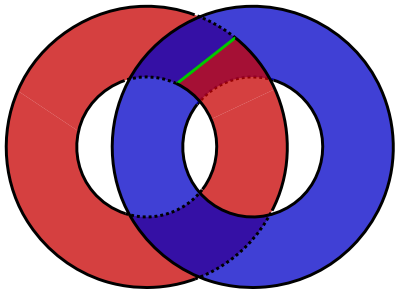

(a) A 2-colored link $L$ admitting a pair of Seifert surfaces intersecting in a ribbon.

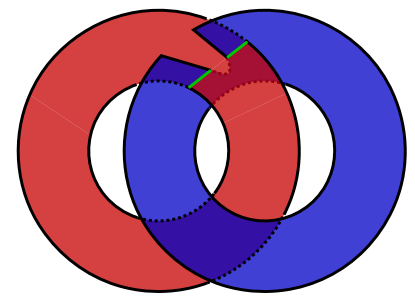

(b) A C-complex $F$ obtained by splitting the ribbon into clasps.

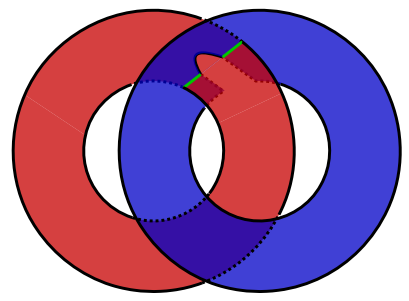

(c) A C-complex $F^{\prime}$ obtained by splitting the ribbon into clasps in a different way.

\section{FigURE 2}

Thus, a correct statement of Lemma 1.1 will require an additional move. Such a move is suggested in Figure 2. These two C-complexes are realized by starting with a pair of surfaces sharing a ribbon intersection and pushing along an arc to split that ribbon into a pair of clasps in two different ways. We introduce a new move which we call (T4) inspired by this example and prove the following.

Theorem 1.3. Let $F$ and $F^{\prime}$ be $C$-complexes bounded by isotopic colored links $L$ and $L^{\prime}$. Then, $F$ and $F^{\prime}$ can be transformed into each other by a finite sequence of the moves (T0), (T1), (T2), (T3), and

(T4) The "push along a different arc" move: Merge two clasps into a ribbon by the inverse of a push along an arc and then push along a different arc, as in Figure 3 .

1.1. Outline of paper. In Section 2, we formally define the notion of a C-complex, prove Theorem 1.2, and present an infinite family of counterexamples to Lemma 1.1. We also pose some questions gauging the degree to which this lemma is false. In Section 3, we assume 


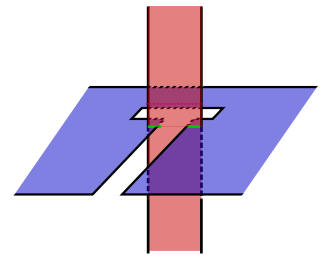

(a)

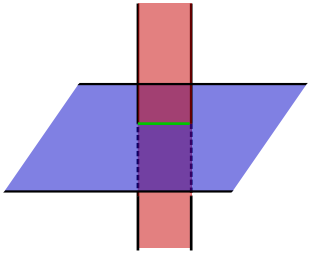

(b)

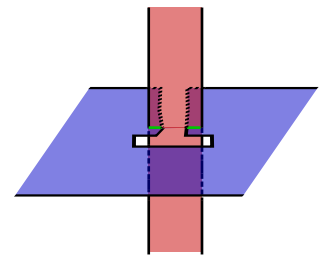

(c)

FiguRE 3. The ribbon intersection of $3 \mathrm{~b}$ may be split into two clasps along any arc running from that ribbon to the boundary $3 \mathrm{a}$ and $3 \mathrm{c}$ give two such choices). The (T4) move allows the replacement of $3 \mathrm{a}$ by $3 \mathrm{c}$.

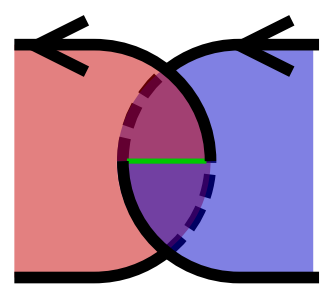

(a) A positive clasp

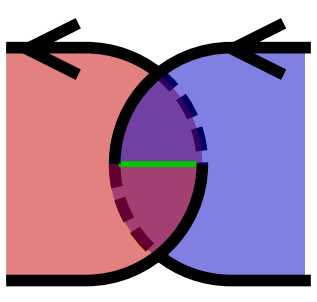

(b) A negative clasp

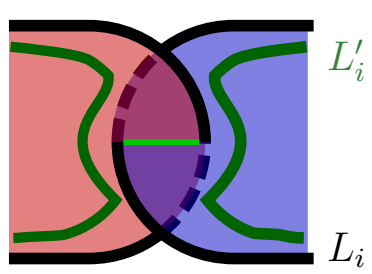

(c) $\operatorname{Skip}(F)$ near a clasp

FiguRE 4

Theorem 1.3 and complete proofs that Cimasoni's geometric construction of the multivariable Conway potential function for colored links does still hold, as does the invariance of the signature and nullity of Cimasoni and Florens [5]. In Section 4, we present a careful, detailed proof of Theorem 1.3 .

1.2. Acknowledgements. The authors would like to thank D. Cimasoni for helpful conversations and encouragement during the progress of this project. They would also like to thank S. Friedl for helpful conversations.

\section{C-Complexes And the COUnterexample to Lemma 1.1}

Definition 2.1. For an $n$-colored link $L=L_{1} \cup \cdots \cup L_{n}$, a $C$-complex for $L$ consists of a union of oriented compact, smoothly embedded surfaces $F=F_{1} \cup \cdots \cup F_{n}$ intersecting transversely in $S^{3}$ so that the following conditions hold:

- Each $F_{i}$ is a Seifert surface with no closed components for the $i$-colored sublink $L_{i} \subseteq L$.

- For any $i \neq j, F_{i} \cap F_{j}$ consists of a disjoint union of embedded arcs each having one endpoint in $\partial F_{i}=L_{i}$ and the other in $\partial F_{j}=L_{j}$. These arcs are called clasps; see Figures $4 \mathrm{a}$ and $4 \mathrm{~b}$.

- There are no triple points; for any $i<j<k, F_{i} \cap F_{j} \cap F_{k}=\emptyset$.

Given a C-complex $F$, one can produce a new link, which we call the skip the clasps link, and denote $\operatorname{Skip}(F)$. In order to do so, let $A_{i} \subseteq F_{i}$ be a collar neighborhood of $\partial F_{i}$ large enough to contain all of the clasps. Observe that $A_{i}$ consists of a disjoint union of embedded annuli $S^{1} \times[0,1]$ bounded by the $i$-colored sublink $L_{i}$ and some other link, $L_{i}^{\prime}$. Set 
$\operatorname{Skip}(F)=L_{1}^{\prime} \cup \cdots \cup L_{n}^{\prime}$. A picture of $\operatorname{Skip}(F)$ near a clasp appears in Figure 4c. We show that $\operatorname{Skip}(F)$ is invariant under the moves (T0), (T1), (T2), and (T3) of Lemma 1.1.

Lemma 2.2. Let $F$ and $F^{\prime}$ be $C$-complexes which differ by a finite sequence of the moves of Lemma 1.1. Then, $\operatorname{Skip}(F) \cong \operatorname{Skip}\left(F^{\prime}\right)$.

Proof. An ambient isotopy (T0) changes $\cup_{i} A_{i}$, and in particular $\operatorname{Skip}(F)$, by an isotopy. A handle attachment (T1) is performed away from a collar neighborhood of the boundary. Thus, it preserves $\cup_{i} A_{i}$ and so $\operatorname{Skip}(F)$. The move (T2) along with its effect on $\operatorname{Skip}(F)$ appears in Figure 5. By inspection, (T2) preserves the isotopy class of $\operatorname{Skip}(F)$. Similarly in Figure 6, we see that (T3) preserves $\operatorname{Skip}(F)$.

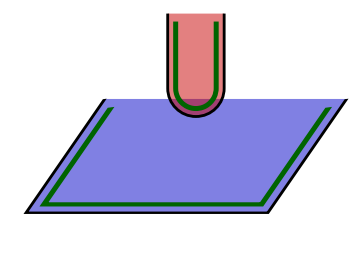

(a) $F$ and $F^{\prime}$ related by a (T2) move

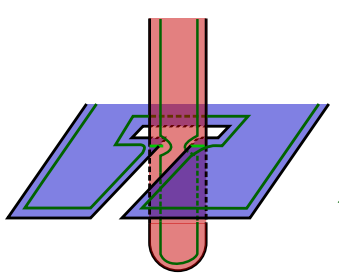

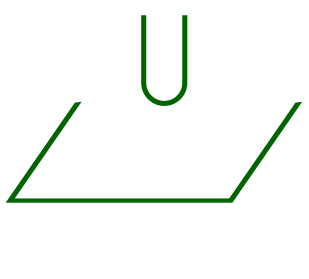

(b) $\operatorname{Skip}(F)$ and $\operatorname{Skip}\left(F^{\prime}\right)$

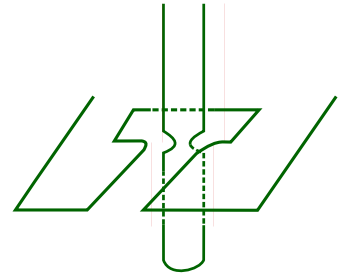

Figure 5. $\operatorname{Skip}(F)$ is preserved by the (T2) move.

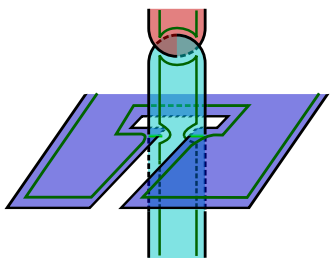

(a) $F$ and $F^{\prime}$ related by a (T3) move

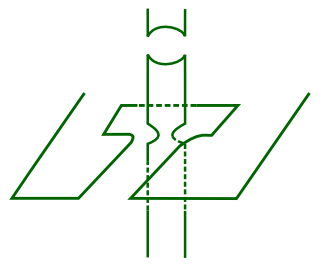

(b) $\operatorname{Skip}(F)$ and $\operatorname{Skip}\left(F^{\prime}\right)$
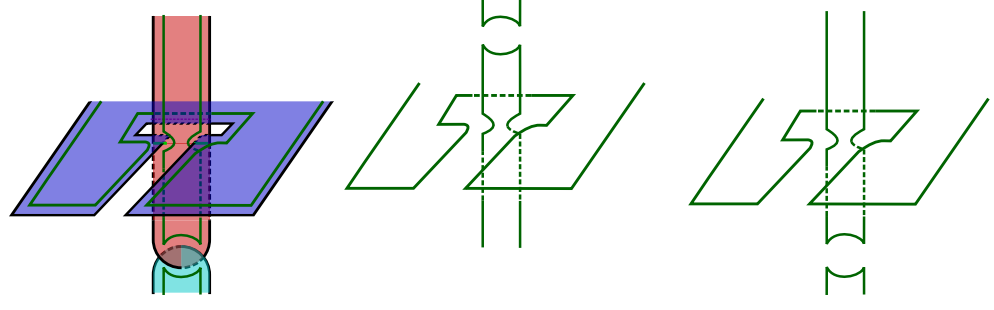

Figure 6. $\operatorname{Skip}(F)$ is preserved by the (T3) move.

As a consequence of Lemma 2.2, if Lemma 1.1 were true, then $\operatorname{Skip}(F)$ would depend only on the link bounded by $F$; $\operatorname{Skip}(F)$ would be be an invariant of colored links. We use this idea to present a counterexample to Lemma 1.1 .

Proof of Theorem 1.2. Consider the C-complexes $F$ and $F^{\prime}$ of Figure 2. In Figure 7 we see $\operatorname{Skip}(F)$ and $\operatorname{Skip}\left(F^{\prime}\right)$. As $\operatorname{Skip}(F)$ has non-vanishing linking numbers and $\operatorname{Skip}\left(F^{\prime}\right)$ is the unlink, Lemma 2.2 implies that $F$ and $F^{\prime}$ are not related by any sequence of the moves from Lemma 1.1.

Example 2.3. In order to evidence that counterexamples to Lemma 1.1 abound, we present an infinite family. Let $K$ be any knot and $L=L_{1} \cup L_{2}=B D(K)$ be the Bing double of $K$ realized as a 2-colored link. In Figure 8 we see a C-complex $F=F_{1} \cup F_{2}$ for $L$, which satisfies that $\operatorname{Skip}(F)$ is the 2-component unlink. On the other hand, $L=B D(K)$ is a boundary link, see for example [3], and so $L$ admits a C-complex $F^{\prime}=F_{1}^{\prime} \cup F_{2}^{\prime}$ with no clasps. Therefore, 

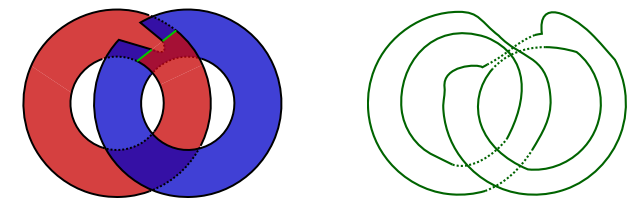

(a) A C-complex $F$ for which $\operatorname{Skip}(F)$ is not the unlink.
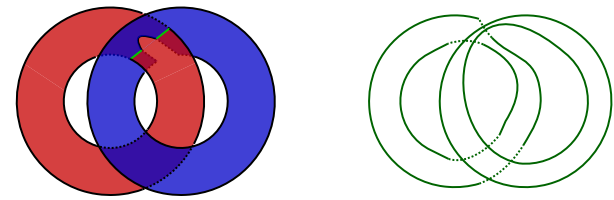

(b) A C-complex $F^{\prime}$ with for which $\operatorname{Skip}\left(F^{\prime}\right)$ is the unlink.

Figure 7. Two C-complexes bounded by the same link with different skip the clasps links.

the collar neighborhood $A^{\prime}$ appearing in the definition of $\operatorname{Skip}\left(F^{\prime}\right)$ is an embedded disjoint union of annuli and parametrizes an isotopy from $B D(K)$ to $\operatorname{Skip}\left(F^{\prime}\right)$. Since $B D(K)$ is not isotopic to the unknot when $K$ is not unknotted, $\operatorname{Skip}(F)$ is not isotopic to $\operatorname{Skip}\left(F^{\prime}\right)$. By Lemma 2.2. $F$ and $F^{\prime}$ are not related by any sequence of moves (T0), (T1), (T2), and (T3).

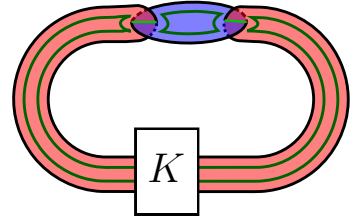

(a)

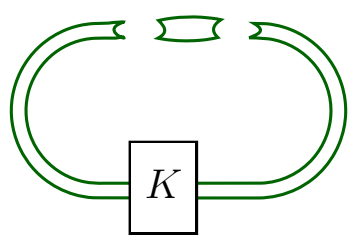

(b)

Figure 8. The Bing double of $K$ admits a C-complex $F$ for which $\operatorname{Skip}(F)$ is the unlink.

We conclude this section with the following questions about the skip the clasps link, justifying further study.

Questions 2.4. For any colored link $L$, set $\operatorname{Skip}(L)=\{\operatorname{Skip}(F) \mid F$ is a C-complex for $L\}$. Does $\operatorname{Skip}(L)$ contain a split link for every $L$ ? More generally, does $\operatorname{Skip}(L)$ contain every colored link $J$ bounding a C-complex with no clasps (known as a colored boundary link) and for which $J_{i}$ is isotopic to $L_{i}$ for all $i$ ? Does there exist a link satisfying that $\operatorname{Skip}(L)$ contains only one link? If $F$ and $F^{\prime}$ are C-complexes for $L$ and $\operatorname{Skip}(F)=\operatorname{Skip}\left(F^{\prime}\right)$ then are $F$ and $F^{\prime}$ related by moves (T0), (T1), (T2) and (T3)?

\section{LinK INVARIANTS FROM C-COMPLEXES}

Lemma 1.1, which we have just seen to be false, is used in the papers [2], [5], and [6] to show that several quantities computed in terms of C-complexes are invariant under moves (T0) through (T3), and thus are colored link invariants. In [2, Lemma 4], this is done for a multivariable polynomial $\Omega_{F}$ associated to a C-complex $F$. This polynomial is then shown to recover the Conway potential function. In [5, Lemma 2.1], this is done for the signature $\sigma_{L}$ and nullity $\eta_{L}$ functions appearing in that paper. In this section, we complete the proofs that $\Omega_{F}, \sigma_{L}$, and $\eta_{L}$ are invariants of colored links by verifying their invariance under the (T4) move. In [6, Section 7] Cimasoni and Turaev introduce a Seifert triple associated to a 
C-complex and use Lemma 1.1 to conclude that it is an invariant of colored links in quasicylinders. While we do not provide the details here, one can check that their Seifert triple is preserved by (T4).

3.1. The multivariable Conway potential function. In [2], Cimasoni provides a formula for the multivariable Conway potential function in terms of the linking numbers of curves sitting on a C-complex. While the fact that this formula gives an invariant of links can be deduced from Hartley's proof that the fact that the multivariable Conway potential function is an invariant [11, one of the goals of [2] is a proof of invariance more in line with the argument of Kauffman [12] of the invariance of the one variable Conway potential function.

We complete Cimasoni's proof of invariance, assuming Theorem 1.3. We begin by recalling Cimasoni's formula for the potential function in terms of a C-complex. Given an $n$-colored link $(L, \sigma)$ bounding a C-complex $F$ and any choice of $\epsilon:\{1, \ldots, n\} \rightarrow\{ \pm 1\}$ one can define a linking matrix analogous to the Seifert matrix. Let $\alpha$ be a curve in $F$ which interacts nicely with the clasps of $F$. In [2] $\alpha$ is called a loop, and the reader is directed to [2], [5], [9] or [10] for a more complete discussion; see in particular [5, Figure 2]. One can then define a pushoff $\alpha^{\epsilon}$ of $\alpha$ by pushing $\alpha$ off of each surface $F_{i}$ in the $\epsilon_{i}$-normal direction. The condition that $\alpha$ is a loop is required to arrange that these pushoffs agree near the clasps. After picking a basis $\left\{\alpha_{i}\right\}$ for $H_{1}(F)$ consisting of loops, the $\epsilon$-linking matrix is the square matrix with $(i, j)$-entry $\left(A_{F}^{\epsilon}\right)_{i, j}=\operatorname{lk}\left(\alpha_{i}, \alpha_{j}^{\epsilon}\right)$. Cimasoni [2] gives a polynomial $\Omega_{F}$ in $n$ variables in terms of these linking matrices and proves that $\Omega_{F}$ recovers the multivariable Conway potential function [2, Lemma 5].

$$
\Omega_{F}\left(t_{1}, \ldots, t_{n}\right)=\operatorname{sgn}(F) \prod_{i=1}^{n}\left(t_{i}-t_{i}^{-1}\right)^{\chi\left(F \backslash F_{i}\right)-1} \operatorname{det}\left(-A_{F}\right) .
$$

Here, $A_{F}=\sum_{\epsilon} \epsilon(1) \cdots \epsilon(n) \cdot t_{1}^{\epsilon(1)} \cdots t_{n}^{\epsilon(n)} \cdot A_{F}^{\epsilon}$ and $\operatorname{sgn}(F)$ is the product of the signs of all clasps of $F$.

Lemma 3.1 (Lemma 4 in [2]). Let $F$ and $F^{\prime}$ be two $C$-complexes for isotopic colored links $(L, \sigma)$ and $\left(L^{\prime}, \sigma^{\prime}\right)$. Then, $\Omega_{F}$ and $\Omega_{F^{\prime}}$ are equal.

Proof. Cimasoni proves that $\Omega$ is preserved by the C-complex moves (T0), (T1), (T2), and (T3). We complete this proof by showing the move (T4) also preserves $\Omega$.

The (T4) move preserves $\operatorname{sgn}(F)$ as well as the Euler characteristics appearing in equation (1). Thus, it suffices to show that $\operatorname{det}\left(-A_{F}\right)=\operatorname{det}\left(-A_{F^{\prime}}\right)$. Without loss of generality, we assume this (T4) move is between $F_{1}$ and $F_{2}$ as in Figure 9 .

We begin with a choice of basis elements for $H_{1}(F)$, taking care to choose a basis including $\alpha_{1}, \alpha_{2}, \alpha_{3}$, and $\alpha_{4}$, as pictured in Figure $9 \mathrm{a}$. We assign the positive side of the surfaces $F_{1}$ and $F_{2}$ to be facing outwards. We note the curve $\alpha_{1}$ is fully contained in the portion of surface $F_{2}$ pictured in Figure 9a. We now compute $A_{F}$ with respect to this basis. 


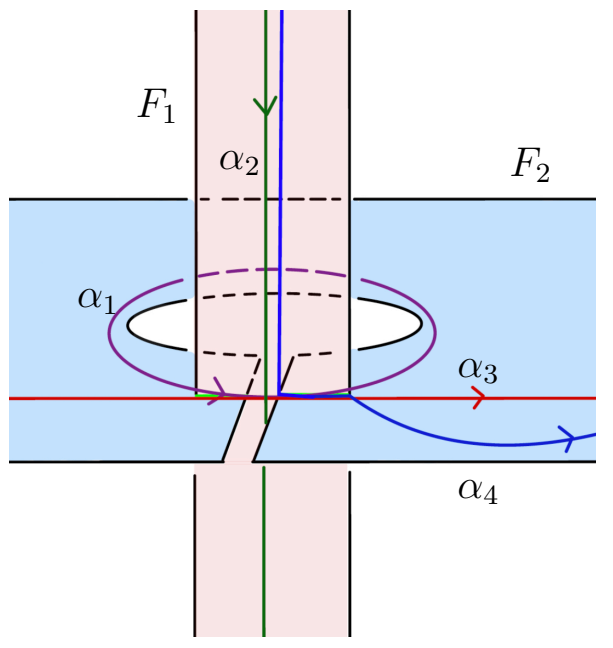

(a) Basis elements for $H_{1}(F)$

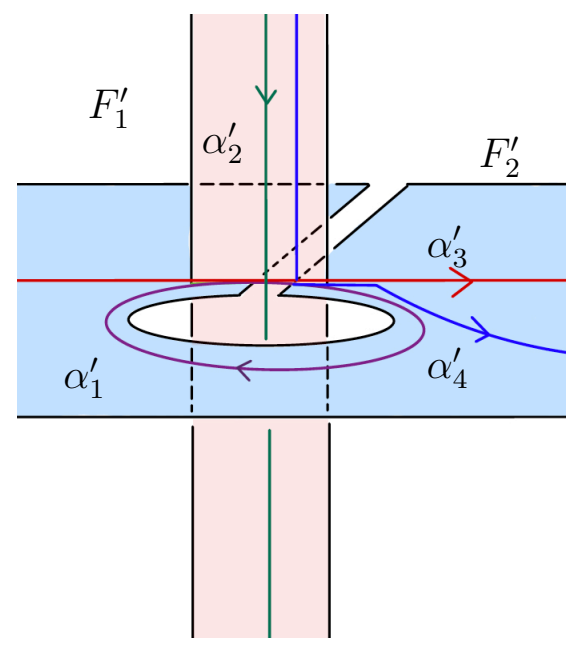

(b) Basis elements for $H_{1}\left(F^{\prime}\right)$

FiguRE 9

$$
A_{F}=\left(\begin{array}{cccc|cccc}
0 & \left(-t_{1} t_{2}+t_{1} t_{2}^{-1}\right) z & 0 & \left(-t_{1} t_{2}\right) z & 0 & 0 & \ldots & 0 \\
\left(-t_{1}^{-1} t_{2}^{-1}+t_{1}^{-1} t_{2}\right) z & a_{22} & a_{23} & a_{24} & & & \\
0 & a_{32} & a_{33} & a_{34} & & B & \\
\left(-t_{1}^{-1} t_{2}^{-1}\right) z & a_{42} & a_{43} & a_{44} & & & \\
\hline 0 & & & & & & \\
0 & & C & & & & & \\
\vdots & & & & & & & \\
0 & & & & & & &
\end{array}\right)
$$

Here, $z=\left(t_{3}-t_{3}^{-1}\right)\left(t_{4}-t_{4}^{-1}\right) \cdots\left(t_{n}-t_{n}^{-1}\right)$. The relationship between first row and column of $A_{F}$ reflects the fact that $\left(A^{\epsilon}\right)^{T}=A^{-\epsilon}$, so that $A_{F}\left(t_{1}, \ldots, t_{n}\right)^{T}=A_{F}\left(-t_{1}^{-1}, \ldots,-t_{n}^{-1}\right)$. To compute $A_{F^{\prime}}$, we use the basis elements for $H_{1}\left(F^{\prime}\right)$ pictured in Figure $9 \mathrm{~b}$.

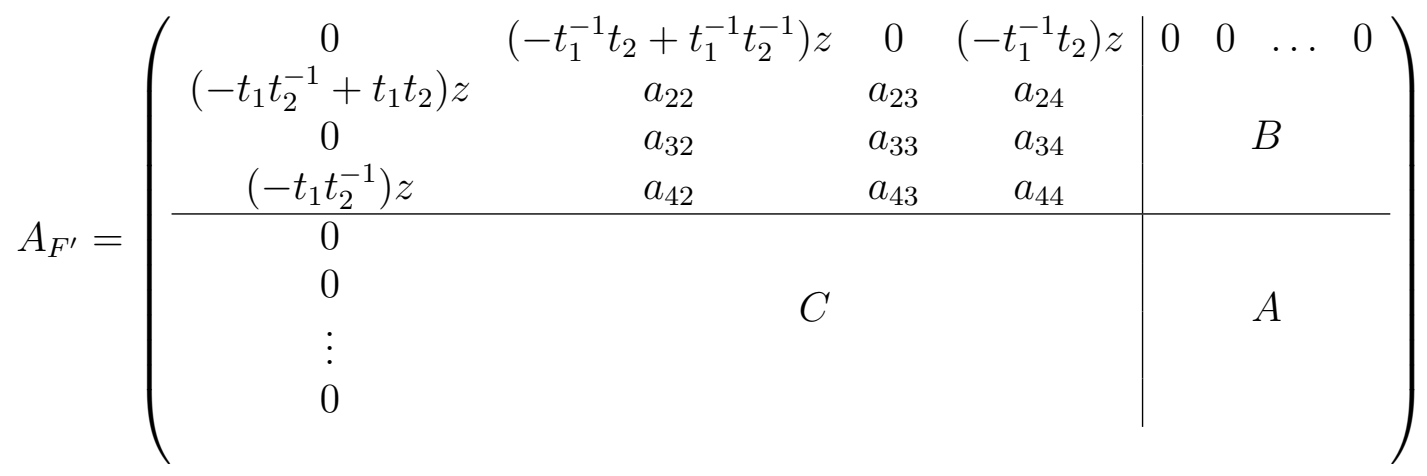

Basic row and column operations show that $\operatorname{det}\left(-A_{F}\right)=\operatorname{det}\left(-A_{F^{\prime}}\right)$; multiplying the first row of $A_{F}$ by $t_{1}^{-2}$ and the first column of $A_{F}$ by $t_{1}^{2}$ results in $A_{F^{\prime}}$. Thus, $\Omega_{F}=\Omega_{F^{\prime}}$.

3.2. The signature and nullity of a colored link. Given an $n$-colored link $(L, \sigma)$, Cimasoni and Florens define the signature and nullity functions of $L[5]$. Let $F$ be a C-complex 
for $L$. For each choice of $\epsilon:\{1, \ldots, n\} \rightarrow\{ \pm 1\}$ and a fixed basis for $H_{1}(F)$, compute the linking matrix $A_{F}^{\epsilon}$ as in subsection 3.1. Given a choice of $\vec{\omega}=\left(\omega_{1}, \ldots, \omega_{n}\right) \in \mathbb{C}^{n}$ such that $\left|\omega_{i}\right|=1$ and $\omega_{i} \neq 1$, for all $i$, define

$$
H_{F}(\vec{\omega})=\sum_{\epsilon}\left(\prod_{i=1}^{n}\left(1-\bar{\omega}_{i}^{\epsilon(i)}\right)\right) A_{F}^{\epsilon} .
$$

The fact that $H_{F}(\vec{\omega})$ is Hermitian follows from the observation that $A_{F}^{-\epsilon}=\left(A_{F}^{\epsilon}\right)^{T}$. Cimasoni and Florens define the signature of $L, \sigma_{L}(\vec{\omega})$, to be the signature of $H_{F}(\vec{\omega})$ and the nullity of $L$ to be $\eta_{L}(\vec{\omega})=\operatorname{null}\left(H_{F}(\vec{\omega})\right)+\beta_{0}(F)-1$. Here, $\beta_{0}$ denotes the zero'th Betti number. They then give the following theorem, which is proved by demonstrating that signature and nullity are preserved by the C-complex moves (T0), (T1), (T2), and (T3) and appealing to Lemma 1.1, which is false. We complete their proof by showing that the move (T4) also preserves signature and nullity.

Theorem 3.2 (Theorem 2.1 of [5]). The signature $\sigma_{L}$ and nullity $\eta_{L}$ do not depend on the choice of C-complex for a colored link L. They are well-defined isotopy invariants of the colored link.

Proof. Let $F$ be a C-complex for an $n$-colored link $L$, and let $F^{\prime}$ be a C-complex related to $F$ by a (T4) move.

Using the basis elements from Figure 9 for $H_{1}(F)$ and $H_{1}\left(F^{\prime}\right)$, and setting $v=(1-$ $\left.\omega_{3}\right) \cdots\left(1-\omega_{n}\right)$, we compute the associated Hermitian matrices $H_{F}(\vec{\omega})$ and $H_{F^{\prime}}(\vec{\omega})$.

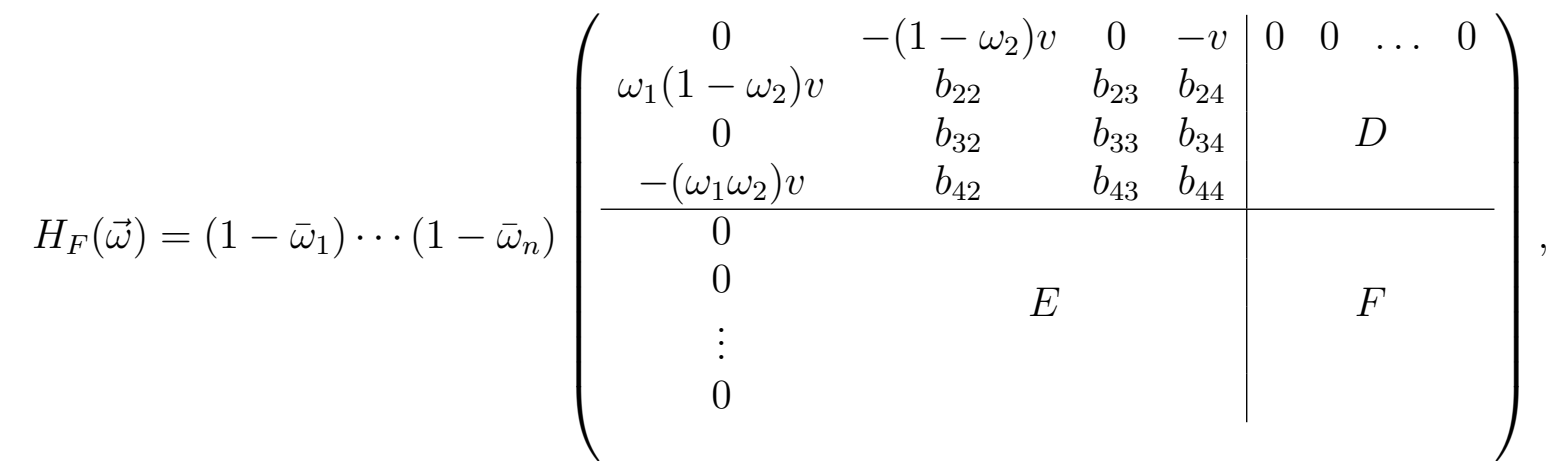

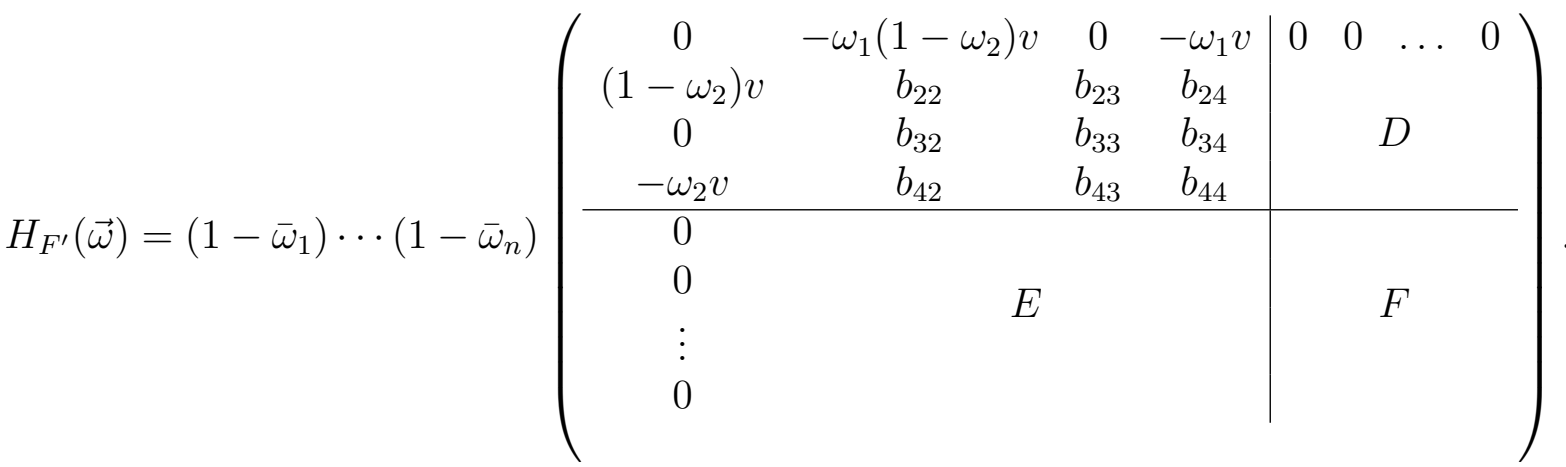

By performing the elementary row and column operations of multiplying the first row of $H_{F}(\vec{\omega})$ by $\omega_{1}$ and the first column of $H_{F}(\vec{\omega})$ by $\bar{\omega}_{1}$, we transform $H_{F}(\vec{\omega})$ into $H_{F^{\prime}}(\vec{\omega})$. This matrix operation preserves the signature and dimension of the nullspace. Further, the (T4) move does not change the number of connected components of the C-complex, so $\beta_{0}(F)=\beta_{0}\left(F^{\prime}\right)$. Thus, $\sigma_{L}(\vec{\omega})$ and $\eta_{L}(\vec{\omega})$ are invariants of the colored link $L$. 


\section{Correcting Lemma 1.1; the Proof of Theorem 1.3}

In this section we prove Theorem 1.3 , thus providing a complete set of moves relating any pair of C-complexes for a fixed link. We start with a general summary of the proof and then spend the bulk of the section on the technical details. We have made an effort to include every detail, even at the expense of brevity; we feel that this choice is appropriate when correcting an error that has gone nearly two decades without detection. Our arguments will pass from C-complexes into the setting of unions of surfaces with more flexibility in their intersections.

Definition 4.1. A union of embedded compact oriented surfaces $F=F_{1} \cup \cdots \cup F_{n}$ with no closed components bounded by the colored link $L=L_{1} \cup \cdots \cup L_{n}$ is called a surface system for $L$ if for all $i, j, k, \ell \in\{1, \ldots, n\}$

$$
\begin{aligned}
\text { I } & : F_{i} \text { is transverse to } F_{j}, \\
\text { II } & : F_{i} \text { is transverse to } L_{j}=\partial F_{j}, \\
\text { III } & : F_{i} \text { is transverse to } F_{j} \cap F_{k}, \\
\text { IV } & : F_{i} \cap \partial\left(F_{j} \cap F_{k}\right)=\emptyset, \\
\text { V } & : F_{i} \cap F_{j} \cap F_{k} \cap F_{\ell}=\emptyset .
\end{aligned}
$$

For any surface system $F=F_{1} \cup \cdots \cup F_{n}$, the stated transversality implies that for any $i \neq j, F_{i} \cap F_{j}$ is a 1-manifold. Therefore any component of $F_{i} \cap F_{j}$ has one of three types of intersection: clasps, ribbons, and circles.

- A component $\alpha$ of $F_{i} \cap F_{j}$ is a clasp intersection if $\alpha$ is an arc with one endpoint in $\partial F_{i}$ and the other in $\partial F_{j}$; see Figure 4 .

- A component $\alpha$ of $F_{i} \cap F_{j}$ is a ribbon intersection if $\alpha$ is an arc with both endpoints in $\partial F_{i}$ or both endpoints in $\partial F_{j}$; a ribbon intersection appears in Figure $3 \mathrm{~b}$.

- Otherwise a component $\alpha$ of $F_{i} \cap F_{j}$ is a circle interior to each of $F_{i}$ and $F_{j}$.

For any distinct $i, j$, and $k, F_{i} \cap F_{j} \cap F_{k}$ is a finite set of points. Such a point is called a triple point of $F$.

Let $F$ be a smooth compact oriented surface. By an isotopy $\Phi^{t}: F \rightarrow S^{3}$ we mean a smooth map $\Phi: F \times[0,1] \rightarrow S^{3}$ so that $\left.\Phi\right|_{F \times\{t\}}$ is a smooth embedding for all $t$. We will use $\Phi^{t}(x)$ to denote $\Phi(x, t)$ and $F^{t}$ to denote $\Phi^{t}(F)$. An isotopy from a (compact, oriented) surface $F$ to $F^{\prime}$ in $S^{3}$ is an isotopy $\Phi^{t}: F \rightarrow S^{3}$ where $\Phi^{0}: F \rightarrow S^{3}$ is the inclusion map and $\Phi^{1}(F)=F^{\prime}$.

Let $F=F_{1} \cup \cdots \cup F_{n}$ and $F^{\prime}=F_{1}^{\prime} \cup \cdots \cup F_{n}^{\prime}$ be C-complexes, or more generally surface systems. For $i=1, \ldots, n$ let $\Phi_{i}: F_{i} \times[0,1] \rightarrow S^{3}$ be an isotopy from $F_{i}$ to $F_{i}^{\prime}$. If the union of restrictions of the various $\Phi_{i}$ to $\partial F_{i}$ gives an isotopy of colored links, then we call $\Phi=\left\{\Phi_{i}\right\}=\left\{\Phi_{1}, \ldots, \Phi_{n}\right\}$ a collection of isotopies from $F$ to $F^{\prime}$ and we will denote by $F^{t}$ the union of surfaces $F_{1}^{t} \cup \cdots \cup F_{n}^{t}$ where $F_{i}^{t}=\Phi_{i}^{t}\left(F_{i}\right)$.

Given such a collection of isotopies, any $t \in[0,1]$ for which $F^{t}$ fails to be a surface system is called a critical time. A perturbation of $\Phi$ allows us to arrange that there are only finitely many critical times and that at any critical time there is a unique critical point at which exactly one of the conditions of Definition 4.1 fails. For $A \in\{\mathbb{I},[\mathrm{II}, \mathrm{III}, \mathrm{IV}, \mathrm{V}\}$, we say a critical point is of type $A$ if the corresponding condition of a surface system fails.

We are now ready to provide an outline of the proof of Theorem 1.3. During this outline we will make reference to several facts. Afterwards, we provide precise statements and proofs. 
Proof of Theorem 1.3. Suppose $F=F_{1} \cup F_{2} \cup \cdots \cup F_{n}$ and $F^{\prime}=F_{1}^{\prime} \cup F_{2}^{\prime} \cup \cdots \cup F_{n}^{\prime}$ are Ccomplexes for two isotopic colored links, $L$ and $L^{\prime}$. We need to exhibit a sequence of moves (T0) through (T4) starting at $F$ and ending at $F^{\prime}$. After some handle additions staying in the category of C-complexes, we arrange that $F_{i}$ is isotopic to $F_{i}^{\prime}$ for all $i=1, \ldots, n$ (Lemma 4.2). In other words, after changing $F$ and $F^{\prime}$ by a collection of (T0) and (T1) moves, there exists a collection of isotopies, $\Phi$ from $F$ to $F^{\prime}$. By perturbing $\Phi$, we arrange that $\Phi$ has only finitely many critical times and that at each critical time there is a single critical point. As we shall see in Proposition 4.3 , if $[a, b] \subset[0,1]$ contains no critical times, then there is an ambient isotopy $\Psi: S^{3} \times[a, b] \rightarrow S^{3}$ such that $F^{t}=\Psi\left(F^{a}\right)$ for all $t \in[a, b]$.

In Figure 10 we see a move which replaces $\Phi$ by another sequence of isotopies with one fewer type I critical point. This move is called a push along an arc and is described in Definition 4.4 . Using this move we remove all critical points of type I, III, and V] in Proposition 4.5.

In Proposition 4.6 further modify $\Phi$ to eliminate all circle intersections and organize the remaining type II and IV critical times into pairs $\left\{\left(a_{i}, b_{i}\right)\right\}$ so that for all $t \in\left(a_{i}, b_{i}\right), F^{t}$ contains exactly one ribbon intersection or exactly one triple point but not both. Away from these subintervals, $F^{t}$ is a C-complex. Thus, we need only analyze how such a pair of critical times changes the C-complex. The Type II critical times which increase the number of ribbon intersections appear in Figure 11. Following any one of these with the reverse of another results in a (T2) or (T4) move. The paired type IV critical times result in a sequence of the moves (T0) though (T4); See Figures 13 and 14 . This completes the proof.

The remainder of this section is devoted to filling in the details above.

Lemma 4.2. Let $F=F_{1} \cup \cdots \cup F_{n}$ and $F^{\prime}=F_{1}^{\prime} \cup \cdots \cup F_{n}^{\prime}$ be $C$-complexes for a link $L$. There exist $C$-complexes $G=G_{1} \cup \cdots \cup G_{n}$ and $G^{\prime}=G_{1}^{\prime} \cup \cdots \cup G_{n}^{\prime}$ for $L$ so that $F$ and $F^{\prime}$ are transformed to $G$ and $G^{\prime}$, respectively, by a sequence of (T0) and (T1) moves such that $G_{i}$ is isotopic to $G_{i}^{\prime}$ for each $i=1, \ldots, n$.

Proof. By [9, Chapter 1 Proposition 2.7], for any $i$, there is a third Seifert surface $V_{i}$ obtained from each of $F_{i}$ and $F_{i}^{\prime}$ by a sequence of ambient isotopies and addition of hollow handles. By conjugating by an ambient isotopy the arcs along which the hollow handles are added, we arrange that each sequence consists of all of the handle additions followed by an ambient isotopy. A further isotopy allows us to arrange that these arcs, and so the resulting hollow handles are disjoint from $F_{j}$ and $F_{j}^{\prime}$ for all $j$.

Since the addition of a hollow handle along an arc disjoint from each other component of a C-complex is precisely the (T1) move, this proves the result.

Thus, it suffices to consider C-complexes $F=F_{1} \cup \cdots \cup F_{n}$ and $F^{\prime}=F_{1}^{\prime} \cup \ldots F_{n}^{\prime}$ which are related by a collection of isotopies. This collection of isotopies need not extend to an isotopy of the whole C-complex, and indeed $F^{t}=\Phi^{t}(F)$ may leave the category of C-complexes. The idea of the proof from here on is to restrict how these isotopies change a C-complex, and more generally any surface system. Proposition 4.3 reveals that away from critical times, a collection of isotopies extends to an ambient isotopy. This result is used implicitly in [10, Lemma 5.1] and [2, Lemma 3]. We state it explicitly here and prove it in subsection 4.1.

Proposition 4.3. Let $F=F_{1} \cup \cdots \cup F_{k}$ be a be a surface system. Let $\Phi$ be a collection of isotopies from $F$ to another surface system. Suppose also that for all $t \in[0,1]$ we have 

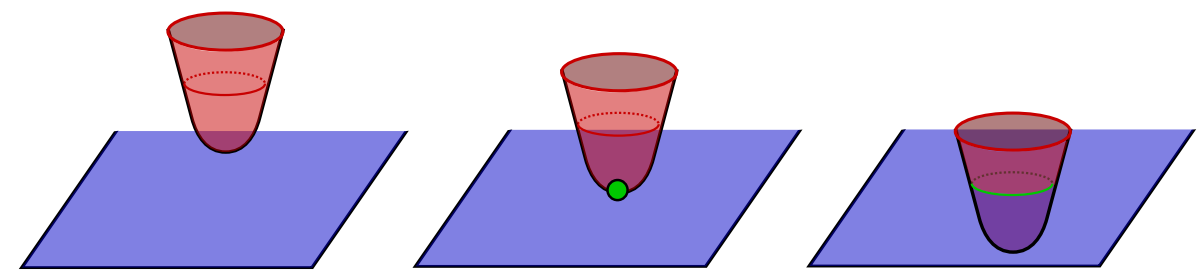

(a) The moment before, during, and after a type I critical point.

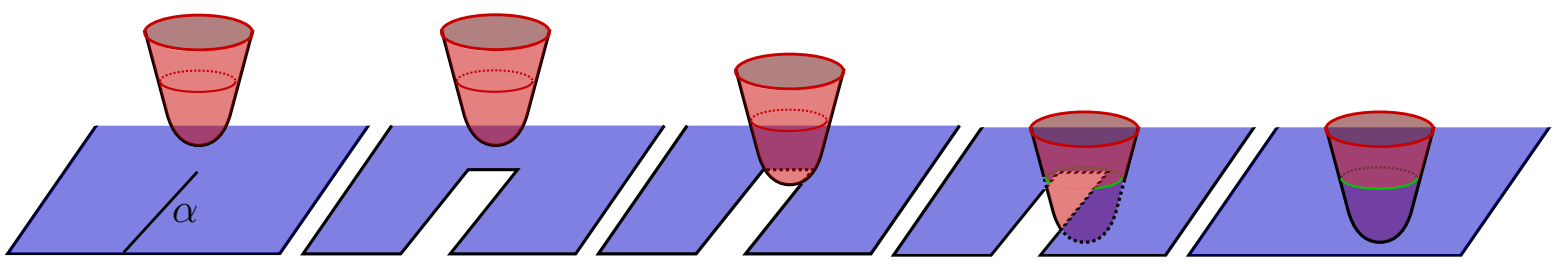

(b) A push along an arc $\alpha$ replaces this type I critical point by type II critical points.

FiguRE 10

that $F^{t}$ is a surface system. Then there is an ambient isotopy $\Psi: S^{3} \times[0,1] \rightarrow S^{3}$ with $\Psi^{t}(F)=F^{t}$.

It remains to control what happens at critical times. We first eliminate critical points of types I, III, and V. A key is the push along an arc move, which we now describe.

Definition 4.4. Let $\Phi$ be a collection of isotopies between surface systems $F$ and $F^{\prime}$. Pick an embedded arc $\alpha \subseteq F_{i}$ with one endpoint in $\partial F_{i}$ and which otherwise is interior to $F_{i}$ as well as an interval $\left[t_{0}, t_{1}\right] \subseteq(0,1)$. Let $U \subseteq F_{i}$ be a regular neighborhood of $\alpha$ disjoint from $\left(\Phi_{i}^{t}\right)^{-1}\left(\partial F_{j}^{t}\right)$ for all $t \in[a, b]$ and all $j \neq i$. Let $r^{t}: F_{i} \rightarrow F_{i}-U$ be an isotopy, supported in a small neighborhood of $U$, with $r^{0}=\operatorname{Id}_{F_{i}}$ and $r^{1}\left(F_{i}\right) \subseteq F_{i}-U$. A new collection of isotopies, $\left(\Phi^{\prime}\right)^{t}$ is given by concatenating $\Phi^{t}$ over $\left[0, t_{0}\right], \Phi^{t_{0}} \circ r^{t}$ over $[0,1], \Phi^{t} \circ r^{1}$ over $\left[t_{0}, t_{1}\right], \Phi^{t_{1}} \circ r^{1-t}$ from $t=0$ to $t=1$, and $\Phi^{t}$ over $\left[t_{1}, 1\right]$. A schematic appears in Figure 10 and an explicit formula appears in (2) below. The push along an arc move replaces $\Phi$ by $\Phi^{\prime}$.

$$
\begin{aligned}
& \left(\Phi^{\prime}\right)_{i}(x, t)= \begin{cases}\Phi_{i}(x, t) & \text { if } 0 \leq t \leq t_{0}-\epsilon \\
\Phi_{i}\left(r^{t}\left(x, \frac{1}{\epsilon}\left(t-t_{0}+\epsilon\right)\right), t_{0}-\epsilon\right) & \text { if } t_{0}-\epsilon \leq t \leq t_{0} \\
\Phi_{i}\left(r^{t}(x, 1), \frac{t_{1}-t_{0}+2 \epsilon}{t_{1}-t_{0}}\left(t-t_{0}\right)+t_{0}-\epsilon\right) & \text { if } t_{0} \leq t \leq t_{1} \\
\Phi_{i}\left(r^{t}\left(x, \frac{-1}{\epsilon}\left(t-t_{1}-\epsilon\right)\right), t_{1}+\epsilon\right) & \text { if } t_{1} \leq t \leq t_{1}+\epsilon \\
\Phi_{i}(x, t) & \text { if } t_{1}+\epsilon \leq t \leq 1\end{cases} \\
& \text { For } j \neq i, \quad\left(\Phi^{\prime}\right)_{j}(x, t)= \begin{cases}\Phi_{j}(x, t) & \text { if } 0 \leq t \leq t_{0}-\epsilon \\
\Phi_{j}\left(x, t_{0}-\epsilon\right) & \text { if } t_{0}-\epsilon \leq t \leq t_{0} \\
\Phi_{j}\left(x, \frac{t_{1}-t_{0}+2 \epsilon}{t_{1}-t_{0}}\left(t-t_{0}\right)+t_{0}-\epsilon\right) & \text { if } t_{0} \leq t \leq t_{1} \\
\Phi_{j}\left(x, t_{1}+\epsilon\right) & \text { if } t_{1} \leq t \leq t_{1}+\epsilon \\
\Phi_{j}(x, t) & \text { if } t_{1}+\epsilon \leq t \leq 1\end{cases}
\end{aligned}
$$

There are some immediate observations. 
- As demonstrated in Figure 10, if $\alpha$ contains a type $\mathbb{1}$ critical point of $\Phi$ at time $t \in\left[t_{0}, t_{1}\right]$ then pushing along $\alpha$ over $\left[t_{0}, t_{1}\right]$ reduces the number of type I critical points by at least one. The push along an arc move can also be used to eliminate critical points of type III and V.

- Pushing along an arc introduces no new critical points of type I, III, or V, although it can introduce critical points of type II and IV.

As a consequence, given any union of isotopies from one surface system to another, we can use the push along an arc move to eliminate all but two types of critical times/points.

Proposition 4.5. Let $F=F_{1} \cup F_{2} \cup \cdots \cup F_{n}$ and $F^{\prime}=F_{1}^{\prime} \cup F_{2}^{\prime} \cup \cdots \cup F_{n}^{\prime}$ be surface systems which are related by a collection of isotopies. There is a new collection of isotopies $\Phi^{\prime}$ from $F$ to $F^{\prime}$ which has only critical points of type II and IV.

Thus, it suffices to explore collections of isotopies between C-complexes which have only critical times of types $[\mathrm{II}$ and $[\mathrm{IV}$ and see how these change the surface system. Using the push along an arc move, we may further control what intersections appear. While we do so formally in Proposition 4.6, we provide an idea here. Since there are no critical points of type $\mathrm{I}$, the only way that a circle intersection can appear is when the two endpoints of a ribbon intersection join together at a type II critical point. By performing a push along an arc crossing this ribbon, we may split this ribbon before that critical time occurs, thus preventing the circle from ever appearing. Therefore, we can avoid circle intersections in a collection of isotopies. While we cannot prevent ribbon intersections from appearing in $F^{t}$, we can split a ribbon intersection into two clasps with a push along an arc as soon as that ribbon appears. Similarly, we may eliminate triple points immediately after they appear. There are two technical difficulties. First, the various circles, ribbons, and triple points in $F^{t}$ may change location as $t$ changes. The intersection we would like to simplify by a push along an arc may escape that arc. Secondly, the various preimage points $\left(\Phi_{i}^{t}\right)^{-1}\left(\partial F_{j}^{t}\right) \subseteq F_{i}$ will also wander about. If one of them passes through the arc we use to push, then the resulting $\Phi^{\prime}$ will no longer restrict to an isotopy on $\partial F$. The following proposition resolves these issues and gives control over the intersections appearing in $F^{t}$.

Proposition 4.6. Let $F$ and $F^{\prime}$ be surface systems related by a collection of isotopies. There exists an additional collection of isotopies $\Phi$ from $F$ to $F^{\prime}$ such that there are disjoint closed subintervals of $[0,1], I_{k}=\left[a_{k}, b_{k}\right]$, for which either $a_{k}$ and $b_{k}$ are both type II critical points or are both type $I V$, and $\Phi$ has no other critical times. In addition, these subintervals satisfy the following conditions:

- For all $t \notin \bigcup I_{k}, F^{t}$ is a $C$-complex.

- For all $t \in I_{k}, F^{t}$ has one ribbon intersection and no triple points or one triple point and no ribbon intersections.

- for all $t \in[0,1], F^{t}$ has no circle intersections.

Proof. Let $\Phi=\left\{\Phi_{i}\right\}$ be a collection of isotopies between C-complexes $F$ and $F^{\prime}$. By Proposition 4.5 we can assume that $\Phi$ has only critical points of type II and IV. Before we can explain how to eliminate circle intersections, we need to explain how to fix a single arc $\beta$ in a circle intersection which persists in $F_{i}^{t} \cap F_{j}^{t}$ for all $t$ in a reasonably long subinterval of $[0,1]$. The argument will require a foray into smooth topology.

Let $\mathbb{F}_{i}=\left\{\left(\Phi_{i}^{t}(x), t\right) \mid x \in F_{i}, t \in[0,1]\right\} \subseteq S^{3} \times[0,1]$ be the trace of the isotopy $\Phi_{i}$. After a small perturbation preserving the property that $\Phi$ is a collection of isotopies we arrange 
that $\mathbb{F}_{i}$ is transverse to $\mathbb{F}_{j}$, and to $\partial \mathbb{F}_{j}$ for all $i \neq j$. We now have that $\mathbb{X}_{i j}:=\mathbb{F}_{i} \cap \mathbb{F}_{j}$ is a smooth 2-manifold in $S^{3} \times[0,1]$. We may further arrange that $\mathbb{F}_{i}$ is transverse to $\mathbb{X}_{j k}$ and $\partial \mathbb{X}_{j k}$. It follows that $\mathbb{X}_{i j k}:=\mathbb{F}_{i} \cap \mathbb{X}_{j k}=\mathbb{F}_{i} \cap \mathbb{F}_{j} \cap \mathbb{F}_{k}$ is a smooth 1-manifold. It is easy to check the following assertions:

(1) $t$ is a type $\mathrm{I}$ critical time with critical point $x \in F_{i}^{t} \cap F_{j}^{t}$ if and only if $(x, t)$ is a critical point for $\left.p\right|_{\mathbb{X}_{i j}}$, the restriction of the projection $p: S^{3} \times[0,1] \rightarrow[0,1]$ to $\mathbb{X}_{i j}$.

(2) $t$ is a type II critical time with critical point $x \in F_{i}^{t} \cap F_{j}^{t}$ if and only if $t \in(0,1)$ and $(x, t)$ is a critical point for $\left.p\right|_{\partial \mathbb{X}_{i j}}$.

(3) $t$ is a type III critical time with critical point $x \in F_{i}^{t} \cap F_{j}^{t} \cap F_{k}^{t}$ if and only if $(x, t)$ is a critical point for $\left.p\right|_{\mathbb{X}_{i j k}}$.

(4) $t$ is a type IV critical time with critical point $x \in F_{i}^{t} \cap F_{j}^{t} \cap F_{k}^{t}$ if and only if $(x, t) \in$ $\partial \mathbb{X}_{i j k}$.

(5) $t$ is a type $\mathrm{V}$ critical time with critical point $x \in F_{i}^{t} \cap F_{j}^{t} \cap F_{k}^{t} \cap F_{\ell}^{t}$ if and only if $(x, t) \in \mathbb{F}_{i} \cap \mathbb{F}_{j} \cap \mathbb{F}_{k} \cap \mathbb{F}_{\ell}$.

Notice that as $\partial \mathbb{X}_{i j k}$ and $\mathbb{F}_{i} \cap \mathbb{F}_{j} \cap \mathbb{F}_{k} \cap \mathbb{F}_{\ell}$ are each 0 -manifolds, any points in these will be critical points for any map to $[0,1]$.

We explain the first of these claims; the rest follow from the same logic. Let $(x, t) \in \mathbb{X}_{i j}$. Since $\mathbb{F}_{i}$ is the trace of an isotopy, there is a tangent vector to $\mathbb{F}_{i}$ at $(x, t), \vec{v}_{i} \in T_{\mathbb{F}_{i}}(x, t)$, with $p_{*}\left(\vec{v}_{i}\right) \neq 0$. Similarly, there is a $\vec{v}_{j} \in T_{\mathbb{F}_{j}}(x, t)$ with $p_{*}\left(\vec{v}_{j}\right) \neq 0$. Since $T_{[0,1]}(t)$ is 1-dimensional, we may normalize $\vec{v}_{i}$ and $\vec{v}_{j}$ so that $p_{*}\left(\vec{v}_{i}-\vec{v}_{j}\right)=0$. This means that $\vec{v}_{i}-\vec{v}_{j} \in T_{S^{3} \times\{t\}}(x, t)$. If $x$ is not a type I critical point, then by the transversality of $F_{i}^{t}$ and $F_{j}^{t}$ in $S^{3}$, there are some $\vec{u}_{i} \in T_{F_{i}^{t} \times\{t\}}(x, t)$ and $\vec{u}_{j} \in T_{F_{j}^{t} \times\{t\}}(x, t)$ so that $\vec{v}_{i}-\vec{v}_{j}=\vec{u}_{i}-\vec{u}_{j}$. Observe that $\vec{v}_{i}-\vec{u}_{i} \in T_{\mathbb{F}_{i}}(x, t), \vec{v}_{j}-\vec{u}_{j} \in T_{\mathbb{F}_{j}}(x, t)$, and $\vec{v}_{i}-\vec{u}_{i}=\vec{v}_{j}-\vec{u}_{j}$. We now see that $\vec{v}_{i}-\vec{u}_{i} \in T_{\mathbb{F}_{i}}(x, t) \cap T_{\mathbb{F}_{j}}(x, t)=T_{\mathbb{X}_{i j}}(x, t)$ and $p_{*}\left(\vec{v}_{i}-\vec{u}_{i}\right)=p_{*}\left(\vec{v}_{i}\right) \neq 0$. We conclude that $(x, t)$ is regular for $\left.p\right|_{\mathbb{X}_{i j}}$.

Conversely, suppose that $(x, t) \in \mathbb{X}_{i j}$ is regular for $\left.p\right|_{\mathbb{X}_{i j}}$. Then there is some $\vec{v} \in T_{\mathbb{X}_{i j}}(x, t)$ with $p_{*}(\vec{v}) \neq 0$. Let $\left\{\vec{x}_{i}, \vec{y}_{i}\right\}$ and $\left\{\vec{x}_{j}, \vec{y}_{j}\right\}$ be bases for $T_{F_{i}^{t} \times\{t\}}(x, t)$ and $T_{F_{j}^{t} \times\{t\}}(x, t)$, respectively. Then $\left\{\vec{v}, \vec{x}_{i}, \vec{y}_{i}\right\}$ and $\left\{\vec{v}, \vec{x}_{j}, \vec{y}_{j}\right\}$ give bases for $T_{\mathbb{F}_{i}}(x, t)$ and $T_{\mathbb{F}_{j}}(x, t)$, respectively. Since $\mathbb{F}_{i}$ and $\mathbb{F}_{j}$ are transverse, $\left\{\vec{v}, \vec{x}_{i}, \vec{y}_{i}, \vec{x}_{j}, \vec{y}_{j}\right\}$ is a generating set for $T_{S^{3} \times[0,1]}(x, t)$. It follows that $\left\{\vec{x}_{i}, \vec{y}_{i}, \vec{x}_{j}, \vec{y}_{j}\right\}$ generates $T_{S^{3} \times\{t\}}(x, t)$, so that $x$ is not a type $\mathrm{I}$ critical point for $F_{i}^{t} \cap F_{j}^{t}$.

Given a generic point $(x, s) \in F_{i}^{s} \cap F_{j}^{s} \times\{s\} \subseteq \mathbb{X}_{i j}$ we may flow in the direction of the gradient of $\left.p\right|_{\mathbb{X}_{i j}}$ to produce a curve $(x(t), t)$ with $t \in\left[t_{0}, t_{1}\right]$ in $\mathbb{X}_{i j}$ running from a local minimum of $\left.p\right|_{\mathbb{X}_{i j}}$ to a local maximum. As $\Phi$ has no Type $\mathrm{I}$ critical points, $\left.p\right|_{\mathbb{X}_{i j}}$ has no critical points. Thus, either $t_{0}=0$ or $\left(x\left(t_{0}\right), t_{0}\right)$ is a type II critical point of $\Phi$. Similarly, either $t_{1}=1$ or $\left(x\left(t_{1}\right), t_{1}\right)$ is a type II critical point. The only type II critical point which result in a local minimum is a birth of a ribbon, as in Figure 11a. The only type [I] critical point which will result in a local maximum is a death of a ribbon (Figure 11a in reverse). Pick $\epsilon>0$ small enough that $\left(t_{0}, t_{0}+\epsilon\right]$ and $\left[t_{1}-\epsilon, t_{1}\right)$ are disjoint and contain no critical times for $\Phi$. The map $y(t)=\left(\Phi_{i}^{t}\right)^{-1}(x(t))$ with $t \in\left[t_{0}+\epsilon, t_{1}-\epsilon\right]$ gives an isotopy of a point interior to $F_{i}$. By the isotopy extension theorem there is an isotopy $\Psi: F_{i} \times[0,1] \rightarrow F_{i}$ such that $\Psi^{t}\left(y\left(t_{0}+\epsilon\right)\right)=y(t)$ for all $t \in\left[t_{0}+\epsilon, t_{1}-\epsilon\right]$. By replacing $\Phi_{i}^{t}$ with $\Phi_{i}^{t} \circ\left(\Psi^{t}\right)$ we arrange that $y(t)$ is constant in time. 


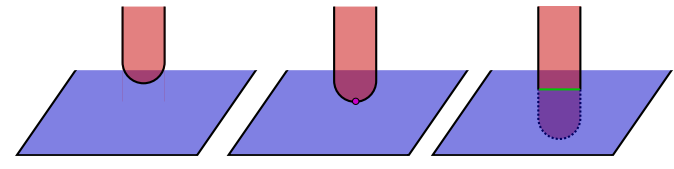

(a) Birth of a ribbon.

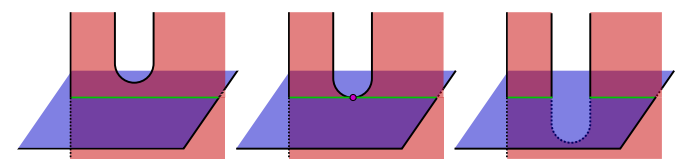

(c) Splitting a clasp into a clasp and a ribbon.

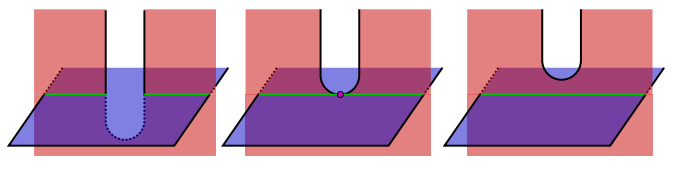

(b) Merging two clasps into a ribbon.

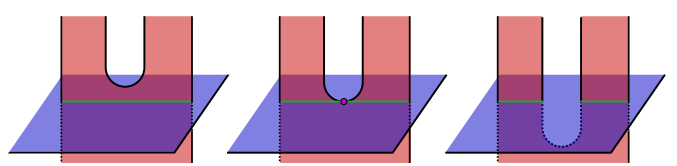

(d) Splitting a ribbon into two ribbons.

Figure 11. The ways that a type II critical point can introduce a ribbon, assuming that a circle never appears.

If we take a generic point $(x, s) \in F_{i}^{s} \cap F_{j}^{s} \times\{s\}$ together with a sufficiently small arc $a \subset F_{i}^{s} \cap F_{j}^{s}$ containing $x$ and follow the argument of the preceding paragraph, then we will produce an arc $\beta$ interior to $F_{i}$ so that $\Phi_{i}^{t}(\beta) \subseteq F_{i}^{t} \cap F_{j}^{t}$ for all $t \in\left[t_{0}+\epsilon, t_{1}-\epsilon\right]$ and $\Phi_{i}^{s}(\beta)=a$. We will refer to this process as fixing the arc $\beta$ over the interval $\left[t_{0}+\epsilon, t_{1}-\epsilon\right]$.

We are now ready to eliminate circle intersections. Let $C \subseteq F_{i}^{s} \cap F_{j}^{s}$ be a circle intersection at time $s$. As above, fix an arc $\beta$ interior to $F_{i}$ over the interval $\left[t_{0}+\epsilon, t_{1}+\epsilon\right]$ so that $\Phi_{i}^{s}(\beta) \subseteq C$ and for all $t \in\left[t_{0}+\epsilon, t_{1}+\epsilon\right], \Phi_{i}^{t}(\beta) \subseteq F_{i}^{t} \cap F_{j}^{t}$. The critical times $t_{0}$ and $t_{1}$ are either 0 or 1 or are births or deaths of ribbons. Thus, for $t$ close to $t_{0}+\epsilon$, or $t_{1}+\epsilon, \Phi_{i}^{t}(\beta)$ is contained in a ribbon or in a clasp. Let $\left[s_{0}, s_{1}\right] \subseteq\left(t_{0}+\epsilon, t_{1}-\epsilon\right)$ be the maximal interval containing $s$ so that $\Phi_{i}^{t}(\beta)$ is contained in a circle intersection for all $t \in\left(s_{0}, s_{1}\right)$. The interval $\left(s_{0}, s_{1}\right)$ can be thought of as the lifespan of $C$. Let $\alpha \subseteq F_{i}$ be an arc running from $\partial F_{i}$ to $\beta$. By picking $\alpha$ so that $\Phi_{i}\left(\alpha \times\left[s_{0}-\epsilon, s_{1}+\epsilon\right]\right)$ is transverse to $\partial \mathbb{F}_{k}^{t}$ for all $k$, we arrange that away from a finite set of times $q_{1}<\cdots<q_{r}, \Phi_{i}^{t}(\alpha)$ is disjoint from $L_{k}$ for all $k \neq i$. By pushing $\alpha$ off itself, we get another arc $\alpha^{\prime}$ disjoint from $\alpha$ so that $\Phi_{i}^{t}\left(\alpha^{\prime}\right)$ is disjoint from $L_{k}$ for all $t \in\left(q_{1}-2 \epsilon, q_{1}+2 \epsilon\right) \cup \cdots \cup\left(q_{r}-2 \epsilon, q_{r}+2 \epsilon\right)$ and all $k \neq i$.

Push along $\alpha$ over $\left[s_{0}-\epsilon, q_{1}-\epsilon\right],\left[q_{1}+\epsilon, q_{2}-\epsilon\right], \ldots,\left[q_{r-1}+\epsilon, q_{r}-\epsilon\right]$, and $\left[q_{r}+\epsilon, s_{1}+\epsilon\right]$ and along $\alpha^{\prime}$ over $\left[q_{1}-2 \epsilon, q_{1}+2 \epsilon\right], \ldots$, and $\left[q_{r}-2 \epsilon, q_{r}+2 \epsilon\right]$. For all $t \in\left[s_{0}, s_{1}\right]$ the circle which contained $\Phi_{i}^{t}(\beta)$ has now been split into either one ribbon or into two ribbons. Repeat this argument until all circle intersections have been removed for all times.

The reader will notice that $\Phi_{i}^{t}(\alpha)$ may contain some points in $F_{i}^{t} \cap F_{j}^{t}$ other than the point where it crosses $\Phi_{i}^{t}(\beta)$. If it does, then we will split more arcs of intersection than we intended to. This will not produce any new circle intersection, and so it is not relevant to the previous steps. It later steps, however, it will be problematic. Fortunately, a similar technique to the paragraph above arranges that for each $t \in[0,1]$, every component of complement in $F_{i}^{t}$ of the intersections with the other components of $F$ contains a point in $\partial F_{i}$. In brief, we push along arcs to reduce the number of components of $F_{i}-\bigcup_{j \neq i}\left(\Phi_{i}^{t}\right)^{-1}\left(F_{j}^{t}\right)$ interior to $F_{i}$.

Now that we have eliminated all circle intersections, we shorten the lifespans of ribbon intersections. We do so by arranging that that at any critical time the number of ribbons either changes from zero to one, from one to zero, or stays zero throughout. Proceeding, let $s$ be a critical time not satisfying these conditions. Then there is a ribbon intersection $R \subseteq F_{i}^{s} \cap F_{j}^{s}$ which persists for all $t \in(s-\epsilon, s+\epsilon)$ for a sufficiently small $\epsilon$. More formally, 
we mean that if we start at a point on $R \times\{s\} \subseteq \mathbb{X}_{i j}$ and flow forward and backwards along the gradient to $\left.p\right|_{\mathbb{X}_{i j}}$ then at least for $t \in(s-\epsilon, s+\epsilon)$ we will see only points $(x(t), t)$ with $x(t)$ sitting on a ribbon intersection in $F_{i}^{t} \cap F_{j}^{t}$. Without loss of generality, we assume that $R$ is interior to $F_{i}^{t}$.

As above we fix an arc $\beta \subseteq F_{i}$ so that $\Phi_{i}^{s}(\beta) \subseteq R$ and so that for some critical times $t_{0}$ and $t_{1}$ and all $t \in\left[t_{0}+\epsilon, t_{1}-\epsilon\right], \Phi_{i}^{t}(\beta) \subseteq F_{i}^{t} \cap F_{j}^{t}$. Recall that $\epsilon$ is picked small enough that $\left(t_{0}, t_{0}+\epsilon\right]$ and $\left[t_{1}-\epsilon, t_{1}\right)$ are disjoint and contain no critical times. Pick a maximal subinterval $\left[s_{0}, s_{1}\right] \subseteq\left[t_{0}+\epsilon, t_{1}-\epsilon\right]$ containing $s$ so that for all $t \in\left(s_{0}, s_{1}\right), \Phi_{i}^{t}(\beta)$ is contained in a ribbon interior to $F_{i}^{t}$.

If $s_{0}=t_{0}+\epsilon$ then set $a_{0}=s_{0}$. Otherwise, $s_{0}$ is a critical time when a ribbon appears, as in Figure 11. In this case set $a_{0}=s_{0}+\epsilon$. Similarly, if $s_{1}=t_{1}-\epsilon$, then set $a_{1}=s_{1}$ and otherwise set $a_{1}=s_{1}-\epsilon$. The interval $\left[a_{0}, a_{1}\right]$ is now an interval contained in the lifespan of the ribbon $r$ we saw at time $s$ and contains all critical times in ribbon's lifespan.

Now we take advantage of the fact that we have arranged that for every $t \in[0,1]$, every component of $F_{i}-\bigcup_{j \neq i}\left(\Phi_{i}^{t}\right)^{-1}\left(F_{j}^{t}\right)$ contains a point of $\partial F_{i}$. By a standard appeal to the Lebesgue number lemma, there exist some $a_{0}=q_{0}<q_{1}<\cdots<q_{n}=a_{1}$ so that for each $\ell=1, \ldots, n$ there is an arc $\alpha_{\ell}$ running from a point on $\partial F_{i}$ to a point on $\beta$ which is otherwise disjoint from $\Sigma_{i}-\bigcup_{j \neq i}\left(\Phi_{i}^{t}\right)^{-1}\left(F_{j}^{t}\right)$ for all $t \in\left[q_{\ell-1}, q_{\ell}\right]$. These $q_{\ell}$ can be chosen so that $\left[q_{\ell}-\epsilon, q_{\ell}+\epsilon\right]$ contains no critical times.

We now change $\Phi$ by pushing along each $\alpha_{\ell}$ over $\left[q_{\ell-1}+\epsilon, q_{\ell}-\epsilon\right]$. Pushing along each of these arcs introduces exactly two new type II critical times, one where a ribbon vanishes and another where it reappears. Moreover, the ribbon containing $\Phi_{i}^{t}(\beta)$ is split into two clasps for all $t \in\left(q_{\ell-1}+\epsilon, q_{\ell}-\epsilon\right)$. We have just reduced by one the number of ribbon intersections persisting at each critical time in $\left(a_{0}, a_{1}\right)$. Iterate this until no ribbon intersection persists at any critical point.

We similarly arrange that no triple point persists at any critical time. The argument is nearly identical to the procedure for controlling ribbon intersections and so we only highlight the differences. Start with a triple point $x \in F_{i}^{s} \cap F_{j}^{s} \cap F_{j}^{s}$ which persists at a critical time $s$. By flowing along the gradient to $\left.p\right|_{\mathbb{X}_{i j k}}$ we fix over an interval a point $y \in F_{i}$ mapping to $x$. A push along a collection of arcs from $\partial F_{i}$ to $y$ reduces the number of triple points which persist at each critical time in the lifespan of the triple point $x$. Depending on the precise implementation of this algorithm, new ribbon intersections may be introduced. The techniques of the preceding paragraphs can now be used to split these into clasps without introducing new triple points.

We have now arranged that during the lifespan of any triple point or any ribbon intersection no other critical times can occur. The existence of the claimed disjoint subintervals follows.

It remains to see how the paired critical points of Proposition 4.6 change the C-complex. Let $a$ be a type II critical time when a ribbon appears. At the corresponding critical point we see one of the the local moves of Figure 11. As a consequence of Proposition 4.6, at no time are there two ribbon intersections, so the move of Figure 11d does not occur. As in Figure 12, the move of Figure 11c can be realized in terms of Figures 11a, and 11b and so we may assume it does not occur. A type II critical time removes a ribbon by reversing these moves. Thus, the effect of the paired critical times of type III is given by following one of 
these by the reverse of another. Following Figure 11a with its own reverse can be obtained by an ambient isotopy. Following Figure $11 \mathrm{a}$ by the reverse of $11 \mathrm{~b}$ results in a (T2) move. Following Figure $11 \mathrm{~b}$ with its reverse results in a (T4) move.

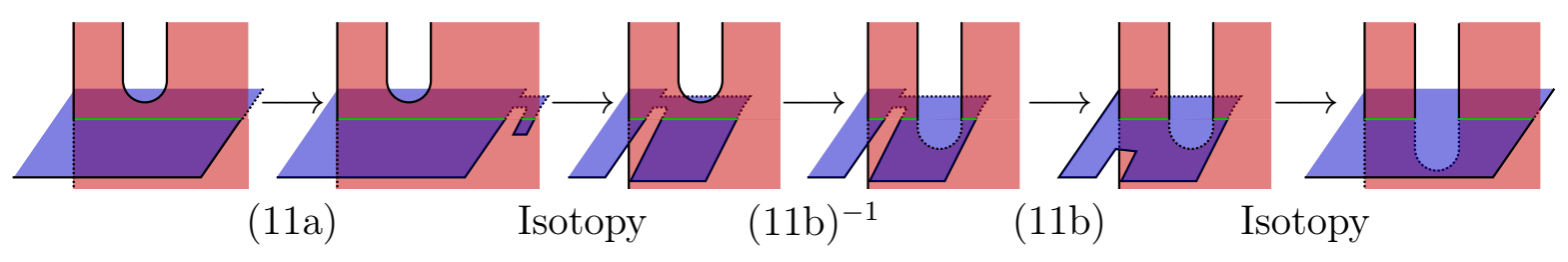

FiguRE 12. The splitting of a clasp into a clasp and a ribbon (Figure 11c) is obtained by the birth of a ribbon (Figure 11a), an ambient isotopy, splitting a ribbon into clasps (the reverse of Figure 11b), merging two clasps into a ribbon (Figure 11b) and finally an ambient isotopy.

Next we analyze any of the the paired type IV critical times $a$ and $b$ of Proposition 4.6 . At time $a$, a point $q \in F_{i}^{a} \cap \partial\left(F_{j}^{a} \cap F_{k}^{a}\right)$ occurs. As $\partial\left(F_{j}^{a} \cap F_{k}^{a}\right) \subseteq \partial F_{j}^{a} \cup \partial F_{k}^{a}$, we assume that $q \in \partial F_{k}^{a}$ without loss of generality. Since $F^{a}$ has only clasp intersections, $q$ is an endpoint of a clasp in $F_{i}^{a} \cap F_{k}^{a}$ and a clasp in $F_{j}^{a} \cap F_{k}^{a}$, and is interior to a clasp in $F_{i}^{a} \cap F_{j}^{a}$. This moment is depicted in Figure 13b. The whole of Figure 13 depicts the moments before this critical time, the lifespan of the resulting a triple point, the critical time $b$ when the triple point dies, and finally the moment after. In Figure 14 we realize the total effect of these paired critical times in terms of (T3) and the moves of Figure 11.

4.1. When does a collection of isotopies extend to an ambient isotopy? The final detail needed to complete the proof of Theorem 1.3 is Proposition 4.3 , which says that away from critical times, a collection of isotopies between pairs of surface systems extends to an ambient isotopy on the whole space. This result seems well-known; however, since we could find no explicit reference, we include an argument for completeness.

Proof of Proposition 4.3. Let $F=F_{1} \cup \cdots \cup F_{n}$ be a surface system and $\Phi=\left\{\Phi_{i}\right\}$ be a collection of isotopies from $F$ to another surface system. Suppose also that for all $t \in[0,1]$ we have that $F^{t}=\Phi_{1}^{t}\left(F_{1}\right) \cup \cdots \cup \Phi_{n}^{t}\left(F_{n}\right)$ is a surface system. Let $\mathbb{F}_{i} \subseteq S^{3} \times[0,1]$ be the trace of $\Phi_{i}^{t}, \mathbb{X}_{i j}=\mathbb{F}_{i} \cap \mathbb{F}_{j}$, and $\mathbb{X}_{i j k}=\mathbb{F}_{i} \cap \mathbb{F}_{j} \cap \mathbb{F}_{k}$.

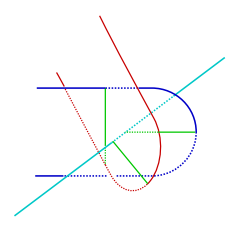

(a)

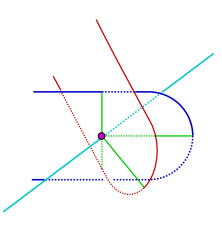

(b)

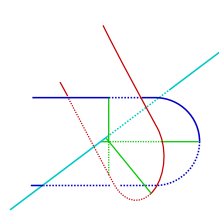

(c)

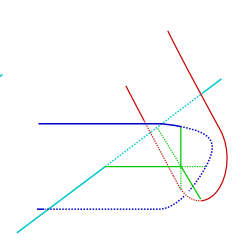

(d)

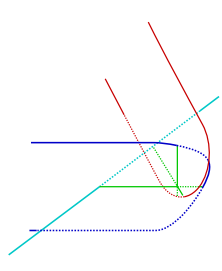

(e)

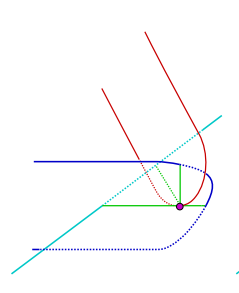

(f)

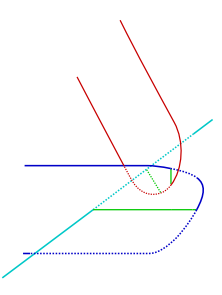

(g)

FiguRE 13. Left to right: A triple point appears at a type IV critical point. The resulting triple point travels the length of a clasp. The triple point vanishes at a second critical time. To aid in readability, the surfaces are not visible. Clasps appear as green. 


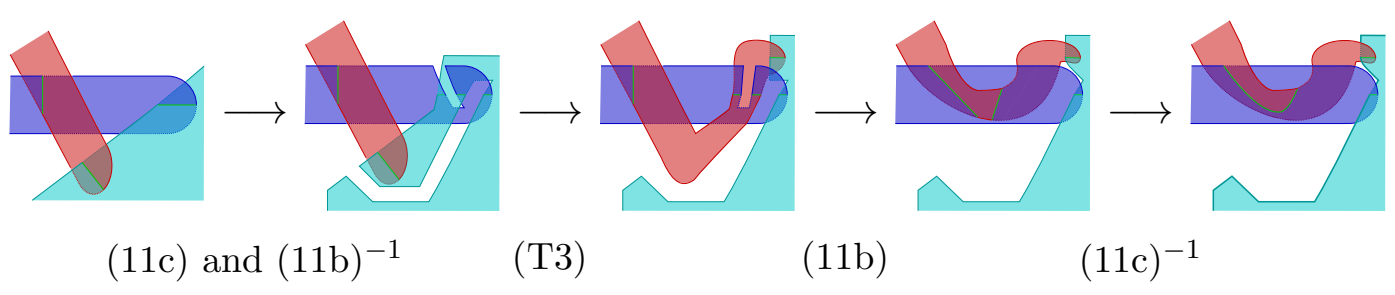

FiguRE 14. A sequence of the moves of Figure 11, their inverses, and the (T3) move relating diagrams 13a and 13g. Transitioning left to right: Split a clasp into a clasp and a ribbon and split that ribbon into two claps; The (T3) move; Merge two clasps into a ribbon; Merge a clasp and a ribbon into a clasp.

As we observed in the proof of Proposition 4.6, critical points of the restriction of $p$ : $S^{3} \times[0,1] \rightarrow[0,1]$ to $\mathbb{X}_{i j}$ and to $\mathbb{X}_{i j k}$ correspond to times when $F^{t}$ fails to be a surface system. We have assumed that this never happens. Thus, $\left.p\right|_{\mathbb{X}_{j k}}$ and $\left.p\right|_{\mathbb{X}_{i j k}}$ have no critical values.

Appealing to the regular interval theorem of morse theory reveals a diffeomorphism $\mathcal{Q}_{i j k}$ : $\left(F_{i} \cap F_{j} \cap F_{k}\right) \times[0,1] \rightarrow \mathbb{X}_{i j k}$ for which $p\left(\mathcal{Q}_{i j k}(x, t)\right)=t$; see [1, Lemma 2.10]. Thus, $\mathcal{Q}_{i j k}$ gives a parametrization of $\mathbb{X}_{i j k}$ as an isotopy of $F_{i} \cap F_{j} \cap F_{k}$. Since there are no quadruple intersections, we have that $\bigcup_{i, j, k} \mathcal{Q}_{i j k}$ is an isotopy of the collection of all triple points. This isotopy extends to an ambient isotopy $\mathcal{R}$ on $S^{3}$.

Replace $\Phi^{t}$ by its composition with the inverse of $\mathcal{R},\left\{\left(\mathcal{R}^{t}\right)^{-1} \circ \Phi_{i}^{t}\right\}_{i}$. We now have that $F_{i}^{t} \cap F_{j}^{t} \cap F_{k}^{t}=F_{i} \cap F_{j} \cap F_{k}$ for all $t \in[0,1]$ and all $i, j, k$. For any triple point $q \in F_{i} \cap F_{j} \cap F_{k}$, take a 3-ball neighborhood $B_{q}$ of $q$, small enough to ensure that some identification $\mathcal{B}^{t}: \mathbb{B}^{3} \rightarrow B_{q}$ sends the $x y$-unit disk to $F_{i}^{t}$, the $x z$-unit disk to $F_{j}^{t}$ and the $y z$-unit disk to $F_{k}^{t}$. The isotopy extension theorem concludes that the isotopy $\mathcal{B}$ extends to an ambient isotopy of $S^{3}$, which we also call $\mathcal{B}$. By replacing $\Phi$ with $\left\{\left(\mathcal{B}^{t}\right)^{-1} \circ \Phi_{i}^{t}\right\}$, we arrange that $F_{i}^{t} \cap B_{p}=F_{i} \cap B_{p}$ for all $t \in[0,1]$ and all $i$.

In the same way, we arrange that $F^{t}$ is fixed in $\nu(\partial F)$, a neighborhood of $\partial F$. Let $U:=$ $\left(\bigcup_{q} B_{q}\right) \cup \nu(\partial F) .\left.\left(\Phi_{i}^{t}\right)\right|_{F_{i} \backslash U}:\left.\left(\Phi_{i}^{t}\right)\right|_{F_{i} \backslash U} \rightarrow S^{3} \backslash U$ is now an isotopy though neat embeddings. The trace is given by $\mathbb{F}_{i}^{\prime}:=\mathbb{F}_{i} \backslash(U \times[0,1])$. They key observation is that we have now removed all triple points.

We may now use the same technique as we did for the triple points to fix the doubleintersections. By applying the regular interval theorem to $\mathbb{F}_{i}^{\prime} \cap \mathbb{F}_{j}^{\prime} \subseteq \mathbb{X}_{i j}$, and then using that there are no triple points we parametrize $\bigcup_{i, j} \mathbb{F}_{i}^{\prime} \cap \mathbb{F}_{j}^{\prime}$ as the trace of an isotopy of $\bigcup_{i, j} F_{i} \cap F_{j} \backslash U$ though neat embeddings into $S^{3} \backslash U$. We extend this isotopy to an ambient isotopy on $S^{3} \backslash U$ and then compose $\left.\left(\Phi_{i}^{t}\right)\right|_{F_{i} \backslash U}$ with the inverse of this ambient isotopy. As a result we ensure that $F_{i}^{t} \cap F_{j}^{t} \backslash U=F_{i} \cap F_{j} \backslash U$ for all $t$. Also similar to the treatment of triple points, we arrange that for a small neighborhood $W \subseteq S^{3} \backslash U$ of $\bigcup_{i, j} F_{i} \cap F_{j} \backslash U,\left(F_{i}^{t} \backslash U\right) \cap W=\left(F_{i} \backslash U\right) \cap W$ for all $t \in[0,1]$ and all $i$.

$\left.\left(\Phi_{i}^{t}\right)\right|_{F_{i} \backslash(U \cup W)}$ is an isotopy through neat embeddedings into $S^{3} \backslash(U \cup W)$ and $\left(\Phi_{i}^{t}\right)\left(F_{i} \backslash(U \cup\right.$ $W))$ is disjoint from $\left(\Phi_{j}^{t}\right)\left(F_{j} \backslash(U \cup W)\right)$ for all $i, j, t$. Thus, $\left.\bigcup_{i}\left(\Phi_{i}^{t}\right)\right|_{F_{i} \backslash(U \cup W)}$ gives an isotopy 
of a disjoint union of neatly embedded surfaces, $\bigcup_{i} F_{i} \backslash(U \cup W)$. This extends to an ambient isotopy on $S^{3} \backslash(U \cup W)$. Extend this ambient isotopy by the identity over $W$ and then over $U$ to arrive at an ambient isotopy of $S^{3}$ that extends each $\Phi_{i}$ simultaneously.

\section{REFERENCES}

[1] Maciej Borodzik, András Némethi, and Andrew Ranicki. Morse theory for manifolds with boundary. Algebr. Geom. Topol., 16(2):971-1023, 2016.

[2] David Cimasoni. A geometric construction of the Conway potential function. Comment. Math. Helv., 79(1):124-146, 2004.

[3] David Cimasoni. Slicing Bing doubles. Algebr. Geom. Topol., 6:2395-2415, 2006.

[4] David Cimasoni, Anthony Conway, and Kleopatra Zacharova. Splitting numbers and signatures. Proc. Amer. Math. Soc., 144(12):5443-5455, 2016.

[5] David Cimasoni and Vincent Florens. Generalized Seifert surfaces and signatures of colored links. Trans. Amer. Math. Soc., 360(3):1223-1264 (electronic), 2008.

[6] David Cimasoni and Vladimir Turaev. A generalization of several classical invariants of links. Osaka J. Math., 44(3):531-561, 2007.

[7] Anthony Conway, Stefan Friedl, and Enrico Toffoli. The Blanchfield pairing of colored links. Indiana Univ. Math. J., 67(6):2151-2180, 2018.

[8] Anthony Conway, Matthias Nagel, and Enrico Toffoli. Multivariable signatures, genus bounds, and 0.5-solvable cobordisms. Michigan Math. J., 69(2):381-427, 2020.

[9] D. Cooper. Signatures of surfaces with applications to knot and link cobordism. $\mathrm{PhD}$ thesis, University of Warwick, 1982.

[10] D. Cooper. The universal abelian cover of a link. In Low-dimensional topology (Bangor, 1979), volume 48 of London Math. Soc. Lecture Note Ser., pages 51-66. Cambridge Univ. Press, Cambridge-New York, 1982.

[11] Richard Hartley. The Conway potential function for links. Comment. Math. Helv., 58(3):365-378, 1983.

[12] Louis H. Kauffman. The Conway polynomial. Topology, 20(1):101-108, 1981.

Department of Mathematics, University of Wisconsin-Eau Claire

Email address: daviscw@uwec.edu

$U R L$ : people.uwec.edu/daviscw

Department of Mathematics, Sam Houston State University

Email address: taylor.martin@shsu.edu

Department of Mathematics, University of Wisconsin-Eau Claire

Email address: ottoa@uwec.edu 